Received: 2019.04.12 Accepted: 2019.05.06 Published: 2019.09 .12
Authors' Contribution: Study Design A Data Collection B Statistical Analysis C Data Interpretation D Manuscript Preparation E Literature Search F Funds Collection G

Corresponding Author: Source of support:

\section{Withaferin-A Inhibits Growth of Drug-Resistant Breast Carcinoma by Inducing Apoptosis and Autophagy, Endogenous Reactive Oxygen Species (ROS) Production, and Inhibition of Cell Migration and Nuclear Factor kappa B (Nf- $\kappa B$ )/Mammalian Target of Rapamycin (m-TOR) Signalling Pathway}

Fifth Department of General Surgery, Second Hospital of Lanzhou University, Lanzhou, Gansu, P.R. China
Background:

Material/Methods:

Results:

Conclusions:

MeSH Keywords:

Full-text PDF:
Ai Lin Song, e-mail: AngieNguyengmu@yahoo.com Departmental sources
The main purpose of this study was to assess in vitro and in vivo the anticancer effect of withaferin-A in human breast carcinoma cells (MDA-MB-231), and to assess its effects on autophagy, cell apoptosis, ROS production, cell migration and invasion, and $\mathrm{Nf}-\mathrm{\kappa B} / \mathrm{m}-\mathrm{TOR}$ signalling pathway.

Proliferation of MDA-MB-231 cells at various doses of the drug was studied by CCK8 cell viability assay. Effects on cell apoptosis were studied by fluorescence microscopy in combination with flow cytometry and Western blot analysis. Effects on autophagy were evaluated by transmission electron microscopy and Western blot. Effects on cellular migration were examined in vitro by wound healing assay.

The results indicated that withaferin-A led to significant reduction of MDA-MB-231 cell viability. The anticancer action of withaferin-A was shown to be due to the stimulation of autophagy, which was accompanied by enhancement of LC3 expression. Withaferin-A prompted mitochondrial apoptosis, which was also associated with increased level of Bax and decreased Bcl-2 in MDA-MB-231 cells. It was also observed that withaferin-A has decreases cellular migration and invasion of the tested human breast cancer cells. The effects of withaferin-A were also investigated in vivo, and it was found that this molecule could inhibit the growth of tumor xenografts in tested mice. Withaferin-A led to suppression of the $\mathrm{Nf}-\mathrm{KB} / \mathrm{m}-\mathrm{TOR}$ signalling pathway. In brief, the withaferin-A molecule has great potential as an anticancer agent against drug-resistant breast cancer, and as such needs to be further studied in detail.

Autophagy • BRCA1 Protein • Breast Neoplasms • Cell Migration Assays • Flow Cytometry

https://www.medscimonit.com/abstract/index/idArt/916931

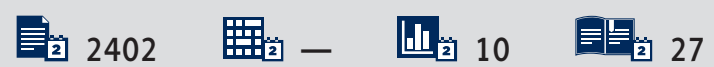




\section{Background}

Breast carcinoma (BC) is the most common cancer among women, and responsible for substantial morbidity and mortality throughout the world [1]. It is the most common cause of death in women, with more than 0.45 million deaths every year [2]. Analysis of the literature shows that breast cancer accounts for about $13.5 \%$ of all cancer-associated deaths in women, with an increasing trend in frequency [3]. The chief problems in the treatment of BC are delayed diagnosis, lack of dependable biomarkers and therapeutic targets, and inadequate availability of effective therapeutic drugs [4,5]. Natural products have a clear advantage over synthetic drugs in that they lack the many serious adverse effects of the latter; therefore, natural products can be useful in the treatment of breast cancer [6]. Plants produces a wide variety of secondary metabolites, including triterpenoids, alkaloids, xanthones, flavonoids, and withanolides. Plant natural products have been used since time immemorial for the treatment of many serious diseases, including cancer, and examples include Taxol, vincristine, vinblastine, and podophyllotoxin. The main characteristic feature of natural plant products is their ability to initiate apoptosis (programmed cell death) within cancer cells $[7,8]$.

Withaferin-A belongs to the steroidal lactone class of naturally occurring plant products, mainly extracted and isolated from Withania somnifera, which is commonly known as "ashwagandha", belonging to the Solanaceae family. It is the firstdiscovered member of a class of natural products with an ergostane-type carbon skeleton, known as withanolides. A wide variety of biological activities have been attributed to withaferin-A, including anticancer activities, immuno-enhancing activities, angiogenesis inhibition effects, cardioprotection, and suppressing cancer cell migration and invasion. Drug resistance in breast cancer is a growing challenge, and many published reports have demonstrated that various natural compounds can inhibit the activity of a membrane protein that is known to pump out anticancer drugs from cells. These compounds can also block specific enzymes that break down drugs, helping to remove of drugs from cells $[9,10]$. The main purpose of the present study was to investigate the anticancer potential of withaferin-A in MDA-MB-231 drug-resistant breast carcinoma cells, as well as to assess its effects on cellular apoptosis and autophagy, endogenous ROS production, cell migration and invasion, and the Nf- $\mathrm{KB} / \mathrm{m}-\mathrm{TOR}$ signalling pathway.

\section{Material and Methods}

\section{Chemicals and other reagents}

Withaferin-A was purchased from Sigma Aldrich. Plasticware used for cell culture experiments was procured from BD
Biosciences. The RPMI-1640 and DMEM (Dulbecco's modified Eagle's medium) were acquired from HyClone (GE Healthcare, Chicago, IL, USA). Penicillin, streptomycin, and fetal bovine serum (FBS) were obtained from Tianjin HaoYang Biological Manufacture Co. (Tianjin, China). Anti-rabbit and anti-mouse secondary antibodies were procured from Cell Signaling Technology, Inc. (Danvers, MA, USA).

\section{Cell culture conditions}

The human breast carcinoma cell line MDA-MB-231 was from the Cancer Research Institute of Beijing (Beijing, China) and preserved in MEM medium (Invitrogen Life Technologies, MA, USA). The cell line was further complemented with $10 \%$ FBS (Invitrogen Life Technologies, MA, USA), $100 \mu \mathrm{g} / \mathrm{ml}$ streptomycin, and $100 \mathrm{U} / \mathrm{ml}$ penicillin G (Himedia, PA, USA) in an incubator at $37^{\circ} \mathrm{C}$ with $5 \% \mathrm{CO}_{2}$.

\section{Cell viability determination by CCK-8 and colony formation assays}

The cell viability of the human breast carcinoma cell line MDAMB-231 was assessed by CCK- 8 assay. MDA-MB-231 cells were seeded in a 96-well plate and were treated with increasing doses of withaferin-A $(0,3.06,6.12,12.5,25,50$, and $100 \mu \mathrm{M})$ at a temperature of $37 \mathrm{C}$ for $24 \mathrm{~h}$. Afterwards, $20 \mu \mathrm{L}$ of CCK- 8 solution was added to the cell culture and incubated for $2 \mathrm{~h}$ in a humidified atmosphere with $5 \% \mathrm{CO}_{2}$ and $95 \% \mathrm{O}_{2}$ at $37^{\circ} \mathrm{C}$. Optical density measurements at $450 \mathrm{~nm}$ wavelength, which is an indication of breast cancer cell viability, were taken using a microplate reader (BD Biosciences, San Jose, CA, USA). Cell morphology of the breast cancer cells after treatment was also examined by phase-contrast microscopy (Nikon Instruments, Inc., NY, USA). The effect of withaferin-A on the formation of human breast cancer cell colonies was assessed as described earlier [11].

\section{Transmission electron microscopy (TEM)}

The MDA-MB-231 human breast cancer cells were subjected to treatment with $0,6,12$, and $24 \mu \mathrm{M}$ doses of withaferin-A. The cells were then subjected to fixation in a solution containing $3 \%$ glutaraldehyde (Sigma Aldrich) and $0.05 \mu \mathrm{M}$ sodium cacodylate, and were then fixed in $1.5 \%$ osmium tetraoxide (OsO4) (Sigma Aldrich) and then dehydrated in alcohol. The MDA-MB-231 human breast cancer cells were then prepared for flat embedding using Epon 812. The cells were finally assessed under an electron microscope (Zeiss CEM 902) (Nikon Instruments Inc., NY, USA).

\section{Western blot analysis}

The protein content of cell lysate was measured by bicinchoninic acid assay (BCA) after the lysis of the MDA-MB-231 human breast cancer cells was carried out in RIPA lysis buffer 

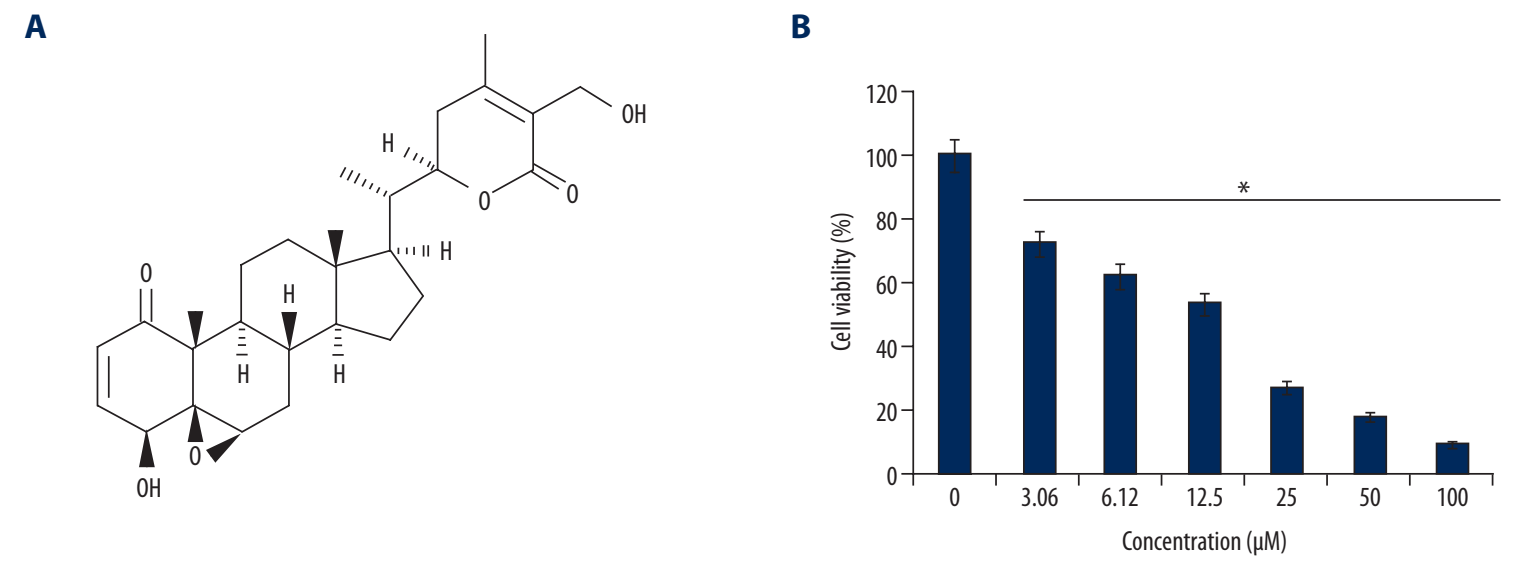

Figure 1. (A) Chemical structure of withaferin-A. (B) Impact of withaferin-A on the viability of the human breast cancer (MDAMB-231) cells as determined by CCK8 assay. The experiment was performed 3 times and results are presented as mean \pm SD $\left({ }^{*} \mathrm{P}<0.01\right)$.

(Invitrogen Life Technologies, MA, USA). SDS-PAGE was used for loading of the samples and the gels were then transported to nitrocellulose membranes. Afterwards, the samples were treated with different primary antibodies for $24 \mathrm{~h}$ at $4^{\circ} \mathrm{C}$. Following this, the membranes were incubated with HRP-conjugated secondary antibody $(1: 1000)$ at $25^{\circ} \mathrm{C}$ for $60 \mathrm{~min}$. Finally, the membranes were treated with enhanced chemiluminescence reagent and the signal was detected using the Odyssey Infrared Imaging System. Actin was used as a control for normalization.

\section{DAPI (4',6-diamidino-2-phenylindole) and annexin V/PI staining}

Using a cell density of $0.6 \times 10^{6}$, MDA-MB-231 human breast cancer cells were cultured in 6-well plates. Following an incubation period of approximately $24 \mathrm{~h}$, the MDA-MB-231 cells were exposed to increasing doses $(0,6,12$, and $24 \mu \mathrm{M})$ of withaferin-A for $24 \mathrm{~h}$ at $37^{\circ} \mathrm{C}$. After sloughing off the cells, $50-\mu \mathrm{L}$ cell cultures were subjected to DAPI staining on glass slides. After covering the glass slides with cover slips, the cells were analyzed under a fluorescent microscope (Nikon Instruments, Inc., NY, USA). For determining percentage of apoptotic cells, Annexin V/PI staining assay using a FACSCalibur flow cytometry (BD Biosciences, NY, USA) was carried out using a previously reported method [12].

\section{Wound healing assay}

Following withaferin-A treatment $(0,6,12$, and $24 \mu \mathrm{M})$ of MDA-MB-231 cells, the medium was taken out and the cancer cells were then washed twice with PBS. A sterile pipette tip was used to scratch a wound in each well, then the cells were washed again and photographed. The cells on plates were cultured for $24 \mathrm{~h}$ and photographed again using an inverted microscope (Leica, Germany).

\section{In vivo study}

Using a xenografted mouse models, the antitumor potential of withaferin-A was assessed in vivo. The 3-week-old male BALB/C nude mice were kept in the animal facility as per the directions and standards provided by the National Institutes of Health for the maintenance and use of the laboratory animals. We subcutaneously injected $5 \times 10^{5}$ MDA-MB-231 cells into the left flanks of the mice. On the first day of the experiment, DMSO (0.1\%)-dissolved withaferin-A diluted with $100 \mu \mathrm{L}$ normal saline at 5, 10, and $20 \mathrm{mg} / \mathrm{kg}$ body weight was injected intraperitoneally into mice $(n=5)$ in each group. Subsequently, withaferin-A was administered to the mice 3 times a week, while DMSO $(0.1 \%)$ in normal saline only was administered to the control mice. At the end of 6 weeks, the mice were sacrificed and tumors were removed for further analysis.

\section{Statistical analysis}

The experiments were performed 3 times. The values presented are means of 3 repeats $\pm S D$. * $p<0.05,{ }^{* *} p<0.01$ and ${ }^{* *} p<0.001$ were considered statistically significant. The $t$ test was performed for statistical analysis using GraphPad Prism 7 software.

\section{Results}

\section{Withaferin-A suppresses the growth of MDA-MB-231 breast cancer cells}

The CCK-8 assay was used to evaluate the effects of withaferin-A (Figure $1 \mathrm{~A}$ ) on the growth of MDA-MB-231 cancer cells at increasing doses. Withaferin-A caused a significant decrease in the proliferation rate of the MDA-MB-231 cells. The effects of 


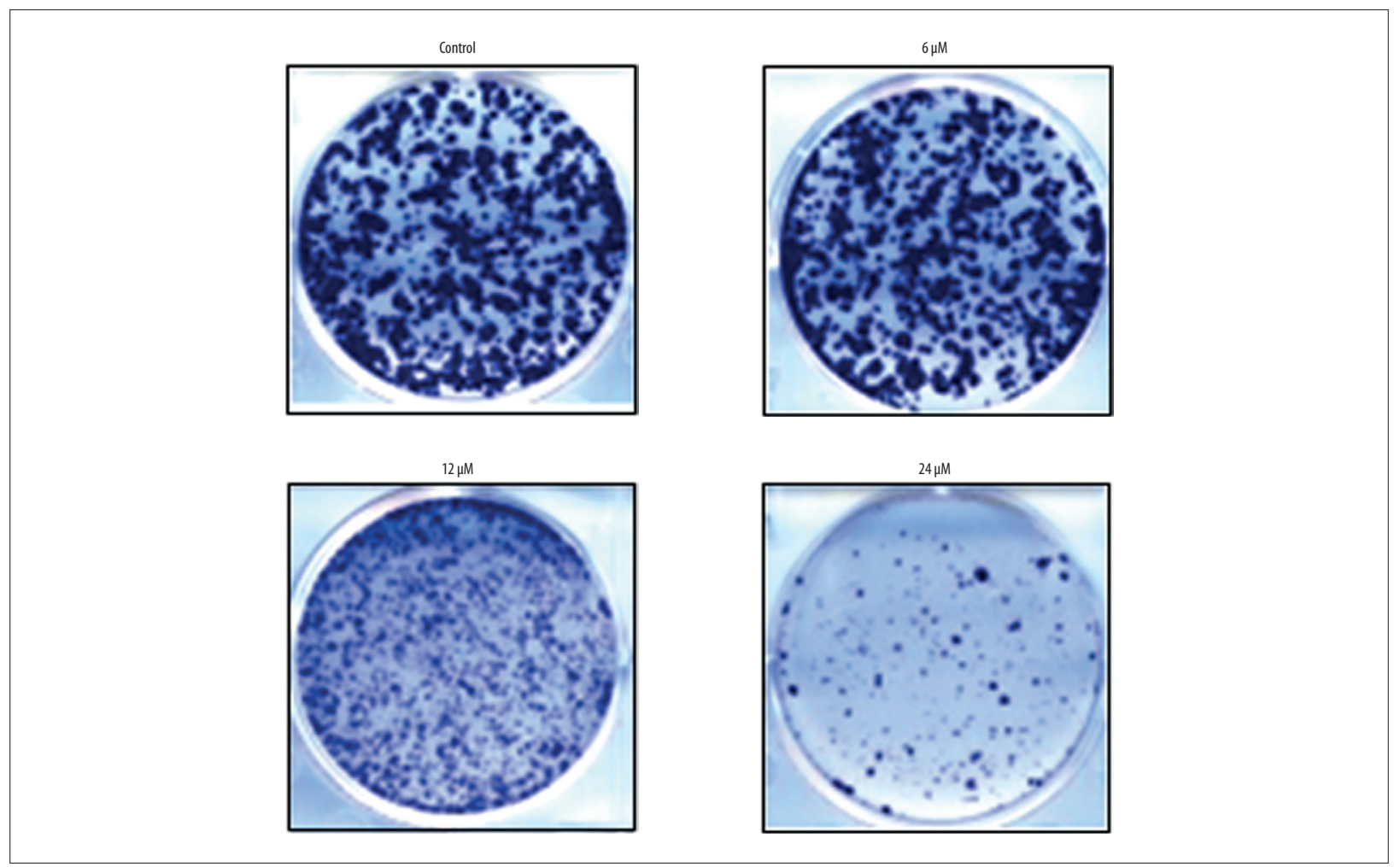

Figure 2. Effects of withaferin-A on cancer cell colony formation in MDA-MB-231 human breast cancer cells. The experiment was performed 3 times.

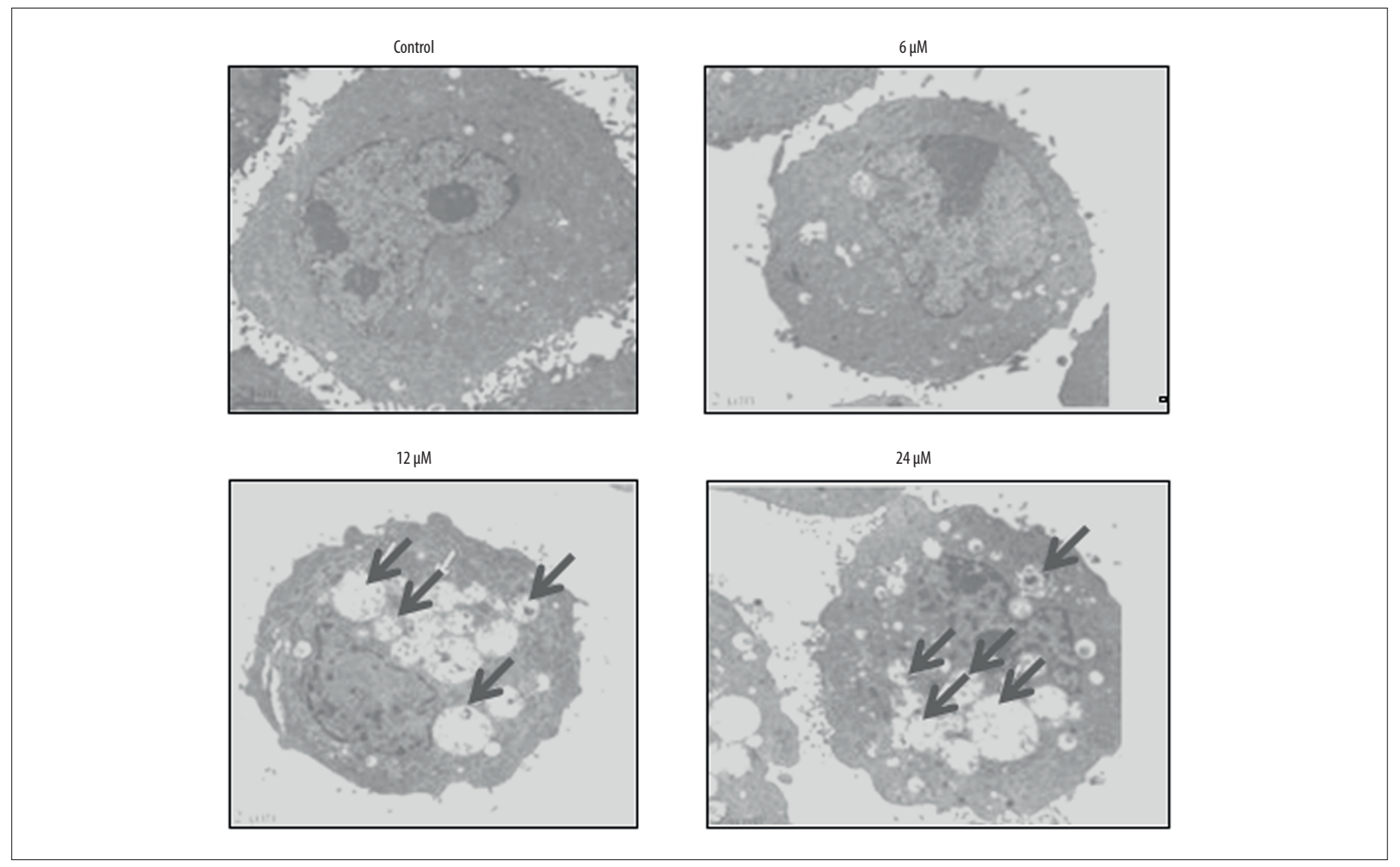

Figure 3. Electron microscopy images of withaferin-A-treated MDA-MB-231 cells showing formation of autophagosomes, thus indicating induction of autophagy. The experiment was performed 3 times. 


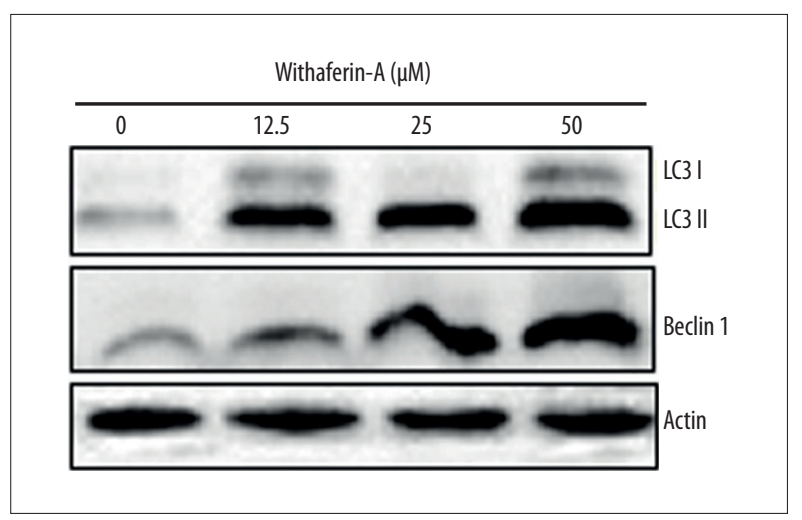

Figure 4. Effect of withaferin-A on autophagy-related protein expression as revealed by Western blot analysis. The experiment was performed 3 times.

withaferin-A on the proliferation rate of the breast cancer cells were concentration-dependent, and the $\mathrm{IC}_{50}$ of $12 \mu \mathrm{M}$ was determined for this molecule against the cancer cells (Figure 1B). Similarly, Figure 2 shows the effect of withaferin-A on the cell colony formation potential of MDA-MB-231 human breast cancer cells, showing that the molecule significantly affected the colony formation ability of these cancer cells.

\section{Control}

$12 \mu \mathrm{M}$
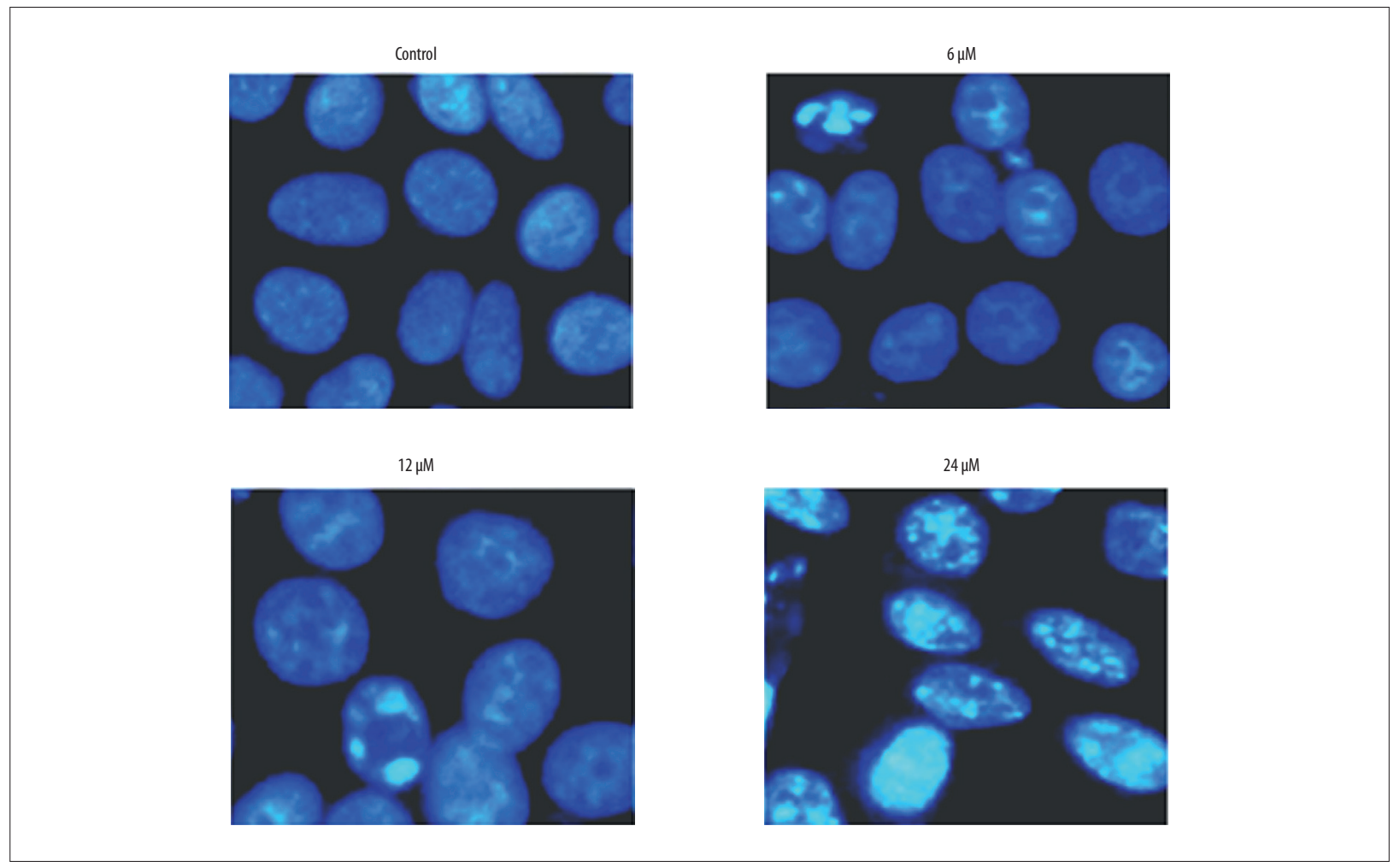

Withaferin-A induces dose-dependent autophagy in MDAMB-231 human breast cancer cells

The autophagic effect of withaferin-A on the MDA-MB-231 cancer cells was investigated by transmission electron microscopy (TEM). The results revealed that withaferin-A initiates the production of autophagosomes (Figure 3) in the MDA-MB-231 cancer cells, indicating that the molecule can induce autophagy. Further, for the confirmation of autophagy, the expression of autophagy-related proteins was investigated, showing that withaferin-A caused an increase of Beclin-1 and LC3-II and suppression of p62 expression. However, no effects were found on the expression of LC3-I (Figure 4). The development of the autophagic vacuoles was observed after drug treatment (Figure 3)

\section{Withaferin-A induces apoptosis in MDA-MB-231 breast cancer cells}

To determine whether the anti-proliferative effects exerted by withaferin-A on the MDA-MB-231 human breast cancer cells are mediated via the induction of apoptotic cell death, DAPI and annexin V/PI staining assays were performed, showing that the percentage of DAPI-positive cells increased significantly, similar to the apoptosis in MDA-MB-231 breast cancer cells (Figure 5). Annexin V/PI staining showed that the breast cancer cell percentage increased in a concentration-dependent

Figure 5. DAPI assay showing induction of apoptosis in MDA-MB-231 cells at indicated concentrations of withaferin-A. The experiment was performed 3 times. 


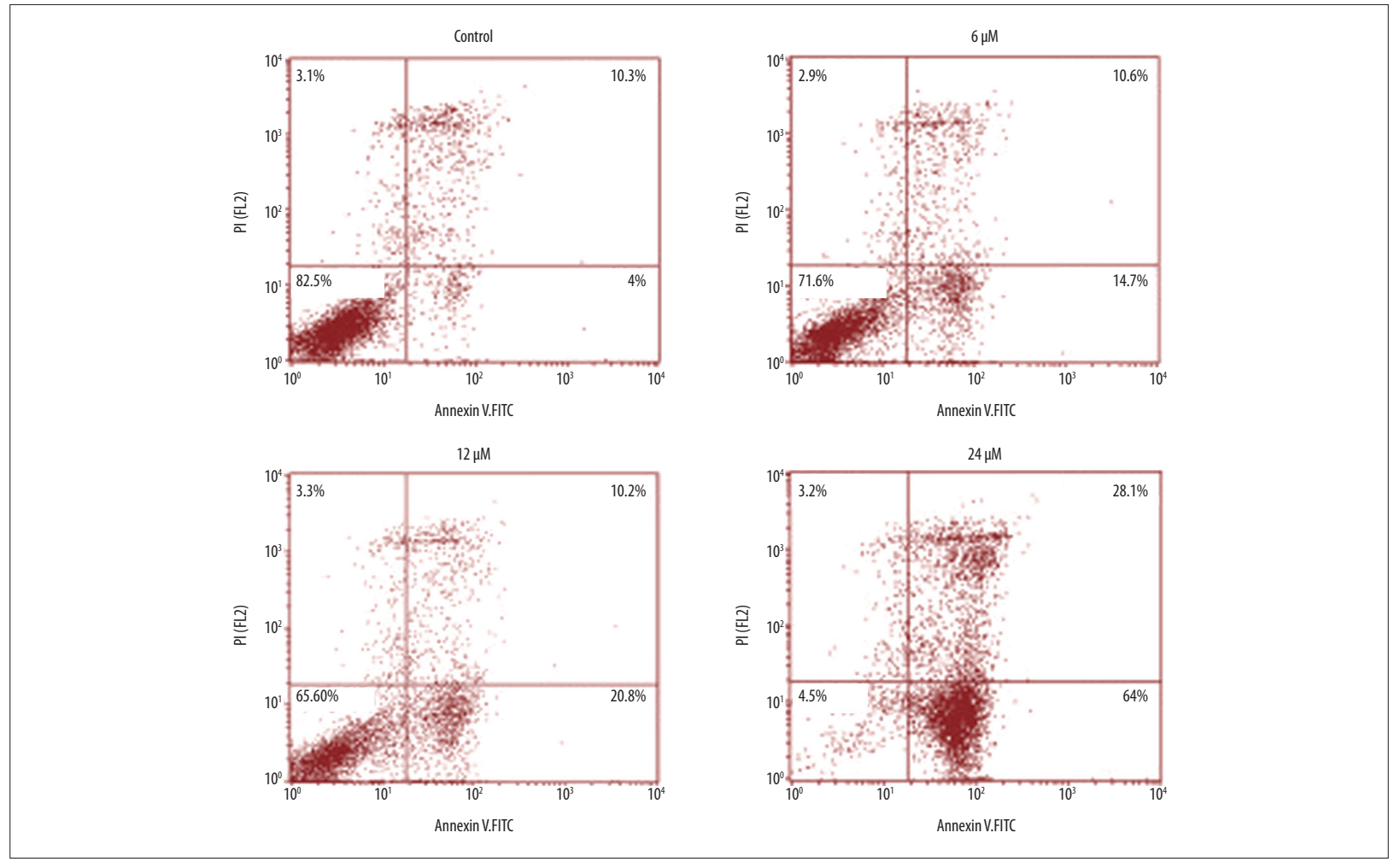

Figure 6. Annexin V/PI assay showing percentage of apoptotic MDA-MB-231 cells at indicated concentrations of withaferin-A. The experiment was performed 3 times.

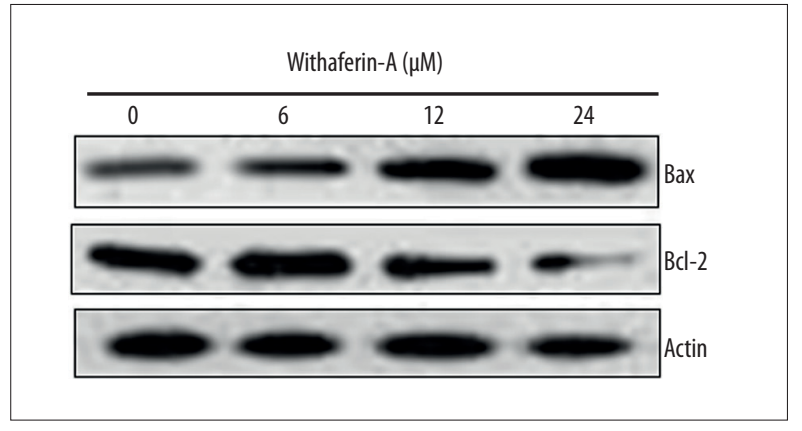

Figure 7. Withaferin-A led to change in the expression of Bax and Bcl-2 as demonstrated by Western blot assay. The experiment was performed 3 times.

manner. The apoptosis percentage increased significantly as the dose of the withaferin-A drug was increased from 0 to 6 , 12 , and $24 \mu \mathrm{M}$ (Figure 6). Further, for the validation of apoptosis, the expression of apoptosis-associated proteins was examined, showing that withaferin-A caused an increase in Bax and decrease in Bcl-2 in MDA-MB-231 cells (Figure 7). These 3 assays (fluorescence microscopy, flow cytometry, and Western blot analysis) clearly showed that withaferin-A induces apoptosis in MDA-MB-231 human breast cancer cells.

\section{Withaferin-A causes inhibition of MDA-MB-231 cell migration}

The in vitro wound healing assay was performed to evaluate whether withaferin-A suppresses the cell migration of human breast cancer cells. The wound healing experiment carried out at various doses of withaferin-A showed that migration of MDA-MB-231 cells was decreased, as evident from the wound width (Figure 8). This result indicates that withaferinA can stop metastasis of these drug-resistant cancer cells under in vitro conditions.

\section{Withaferin-A inhibits tumor growth in vivo}

Since withaferin-A was shown to exert significant anticancer effects on MDA-MB-231 human breast cancer cell lines in vitro, we also examined the anticancer effects of this molecule in vivo in a xenografted mouse models. The results indicated that withaferin- $A$ at the dosage of $20 \mathrm{mg} / \mathrm{kg}$ significantly suppressed the growth of the xenografted tumors. Further, withaferin-A inhibited the tumor weight and volume in a dose-dependent manner (Figure 9A, 9B). 


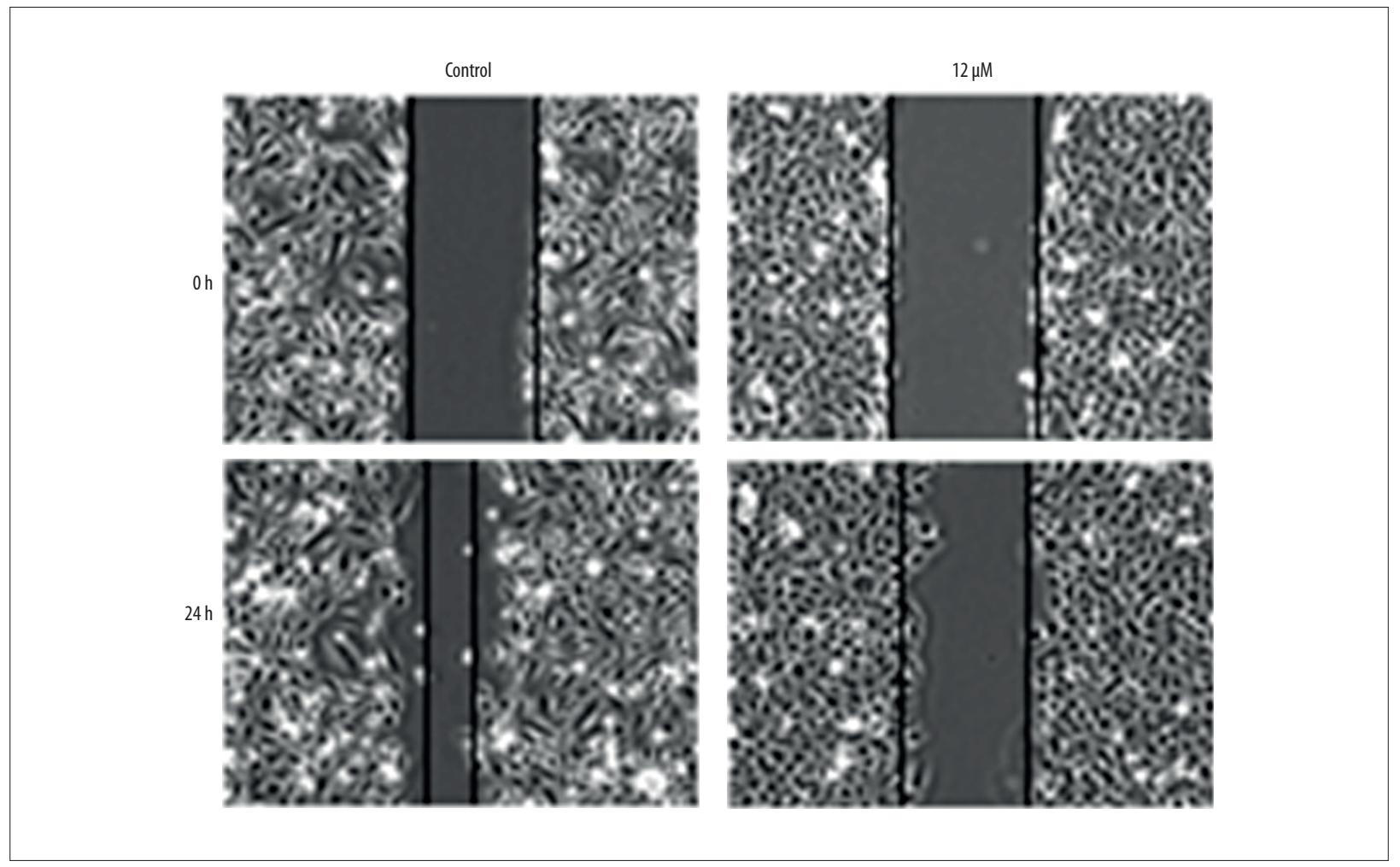

Figure 8. Cell migration in MDA-MB-231 human breast cancer cells is inhibited by withaferin-A in a dose-dependent manner. The experiment was performed 3 times.

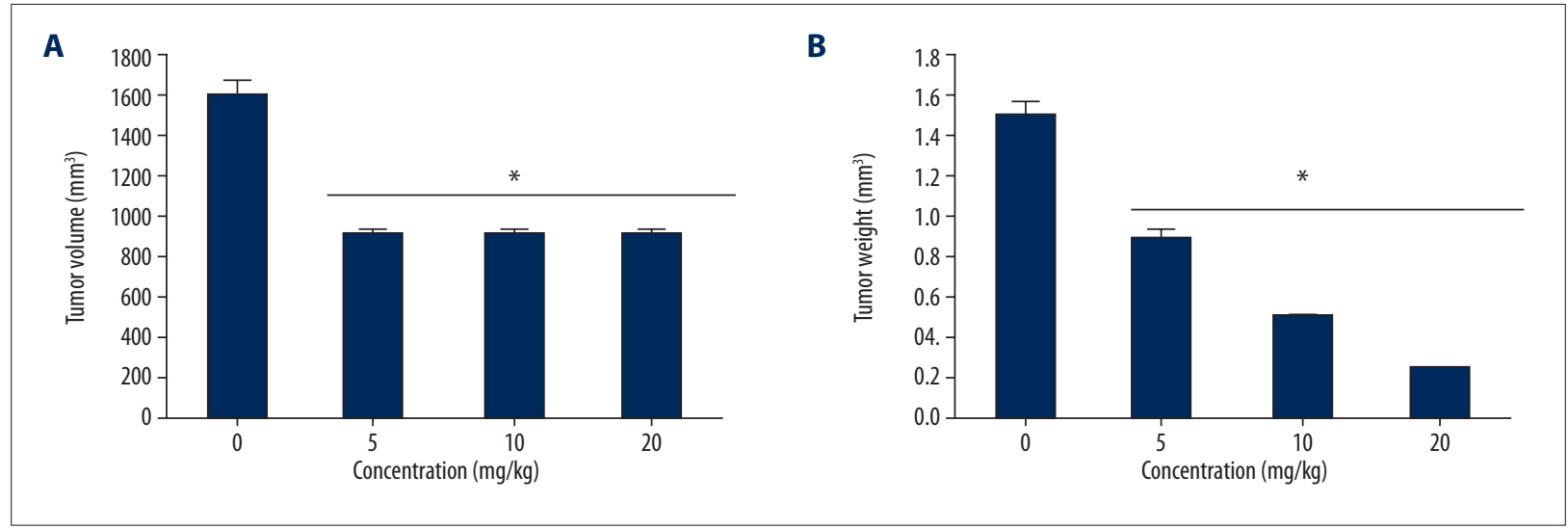

Figure 9. Impact of withaferin-A on xenografted tumor growth. (A) Tumor volume. (B) Tumor weight. The results are shown as mean $\pm S D\left({ }^{*} p<0.01\right)$ and the experiment was performed 3 times.

\section{Withaferin-A inhibits the Nf- $\kappa \mathrm{B}$ signalling pathway}

We also assessed the effects of withaferin-A on the Nf- $\mathrm{KB}$ signalling pathway of MDA-MB-231 human breast cancer cells. We found that withaferin-A caused a significant drop in the expression of $\mathrm{Nf}-\mathrm{KB}$, and these inhibitory effects of withaferin-A exhibited a dose-dependent trend (Figure 10).

\section{Discussion}

Breast carcinoma (BC) is the most commonly diagnosed cancer among women, and is responsible for substantial morbidity and mortality throughout the world. In women, it is the most common cause of death, with more than 0.45 million deaths every year [1,2]. Treatment of cancer includes many approaches, including surgical resection, radiotherapy, and chemotherapy, as well as therapy using lasers, depending on the type of tumor and its stage [13]. Out of these treatment options, 


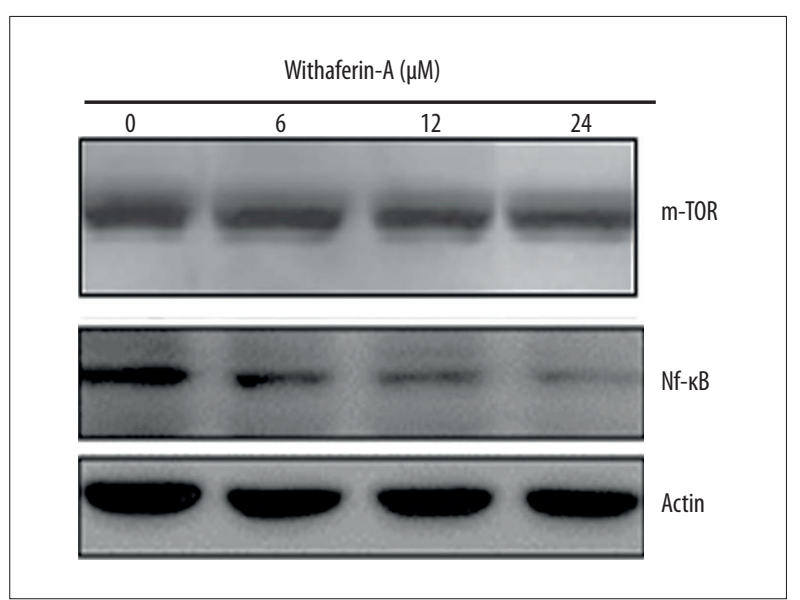

Figure 10. Western blot analysis showing concentrationdependent inhibition of the $\mathrm{Nf}-\mathrm{KB} / \mathrm{m}$-TOR expression in human breast cancer cells upon treatment with varied concentrations of withaferin-A. The results are depicted as mean $\pm S D\left({ }^{*} p<0.01\right)$ and the experiment was performed 3 times.

chemotherapy, is still the most promising option for treating cancer. However, chemotherapy treatment fails in $90 \%$ of cases, and this failure usually occurs during invasion and metastasis of the cancer. Additionally, after administering a particular anticancer drug for a prolonged time, many cancer cells within the patient's body become resistant to the anticancer drug, which further complicates cancer treatment [14]. Drug resistance is a growing problem in the field of cancer chemotherapy, and there is a pressing need to find alternative anticancer drugs which can avert drug resistance acquired by cancer cells. In the present study, we investigated the anticancer effects of withaferin-A, which is a plant withanolide previously shown to have significant anticancer activity. A previous study showed that withaferin-A inhibits the survival of several human and murine B cell lymphoma cell lines [15]. Regarding its in vivo anticancer effects, it has been reported that withaferin-A administration suppressed the growth of Ehrlich ascites cancer in more than $50 \%$ of the treated mice. In another study, the authors reported that a single injected dose of $30 \mathrm{mg}$ withaferin-A/kg body weight significantly prolonged the survival of S-180 ascites-bearing mice $[16,17]$. It has also been reported that withaferin-A causes radiation sensitization in Ehrlich ascites carcinoma-bearing mice, and similar growth inhibition and radiosensitization effects induced by withaferin-A in B16F1 mouse melanoma and fibrosarcoma have been reported $[18,19]$.
The fact that withaferin-A induces apoptosis has been reported previously by many studies. It has been reported to induce apoptosis under both in vitro and in vivo conditions in a number of tumor types, including breast cancer, cervical cancer, leukemia, melanoma, kidney cancer, prostate cancer, and many other cancers [20-22]. Multidrug-resistant breast cancer is a very complicated clinical disease which arises from a wide range of molecular changes, especially changes in the PI3K/AKT signalling pathway. Drug resistance in breast cancer is a growing problem, and this drug resistance can occur in at least a one-quarter of all cases. The incidence of breast cancer drug resistance to anticancer drugs increases with disease progression [23-27].

In the present study, withaferin-A was shown to inhibit growth of MDA-MB-231 human breast cancer cells and to inhibit its cell colony formation ability. Further, it was shown by transmission electron microscopy in combination with Western blot analysis that withaferin-A induces autophagy in these MDAMB-231 drug-resistant breast cancer cells. Also, using fluorescence microscopy with DAPI staining and flow cytometry, we showed that withaferin-A not only induces autophagy, but could also induces apoptosis in these cancer cells. The apoptotic results were further confirmed by Western blot analysis, which showed that withaferin-A increased Bax expression and decreased $\mathrm{Bcl}-2$ expression, in a dose-dependent manner. Effects on cell migration were assessed by wound healing assay, showing that withaferin-A can inhibit MDA-MB-231 cancer cell migration. Finally, in vivo results showed that withaferin-A not only shows in vitro anticancer effects, but also has significant in vivo anticancer activity, inhibiting both tumor mass and volume in a dose-dependent manner.

\section{Conclusions}

Withaferin-A is a potential and promising drug candidate for breast cancer management as shown by the results of the present study, indicating that withaferin-A induces autophagy and apoptosis, inhibits cell migration, and suppresses in vivo tumor growth in a xenografted mouse model.

\section{Conflict of interest}

None. 


\section{References:}

1. DeSantis CE, Ma J, Goding Sauer A et al: Breast cancer statistics, 2017, racial disparity in mortality by state. Cancer J Clin, 2017; 67: 439-48

2. Mariotto $A B$, Etzioni $R$, Hurlbert $M$ et al: Estimation of the number of women living with metastatic breast cancer in the United States. Cancer Epidemiol Prevent Biomarkers, 2017; 26: 809-15

3. Zheng J, Tabung FK, Zhang J et al: Association between post-cancer diagnosis dietary inflammatory potential and mortality among invasive breast cancer survivors in the Women's Health Initiative. Cancer Epidemiol Prev Biomarkers, 2018; 1: 22-27

4. Greenlee H, DuPont, Reyes MJ et al: Clinical practice guidelines on the evidence based use of integrative therapies during and after breast cancer treatment. Cancer J Clin, 2017; 5: 196-201

5. Lee KL, Janz NK, Zikmund-Fisher BJ et al: What factors influence women's perceptions of their systemic recurrence risk after breast cancer treatment? Med Decis Making, 2017; 1: 19-24

6. Shakya AK: Medicinal plants: Future source of new drugs. IntJ Herbal Med, 2016; 4: 59-64

7. Santhosh RS, Suriyanarayanan B: Plants: A source for new antimycobacterial drugs. Planta Medica, 2014; 80: 9-21

8. Howes MJ: The evolution of anticancer drug discovery from plants. Lancet Oncol, 2018; 19: 293-94

9. Kupchan SM, Anderson WK, Bollinger P et al: Tumor inhibitors. XXXIX. Active principles of Acnistus arborescens. Isolation and structural and spectral studies of withaferin A and withacnistin. J Org Chem, 1969; 34(12): 3858-66

10. Mohan R, Hammers HJ, Bargagna-Mohan $P$ et al: Withaferin $A$ is a potent inhibitor of angiogenesis. Angiogenesis, 2004; 7(2): 115-22

11. Borowicz S, Van Scoyk M, Avasarala S et al: The soft agar colony formation assay. J Vis Exp, 2014; (92): e51998

12. Hua F, Li CH, Chen XG, Liu XP: Daidzein exerts anticancer activity towards SKOV3 human ovarian cancer cells by inducing apoptosis and cell cycle arrest, and inhibiting the Raf/MEK/ERK cascade. Int J Mol Med, 2018; 41(6): 3485-92

13. Longley DB, Johnston PG: Molecular mechanisms of drug resistance. J Pathol, 2005; 205(2): 275-92

14. Goodman LS, Wintrobe MM, Dameshek W et al: Nitrogen mustard therapy: Use of methyl-bis (beta-chloroethyl) amine hydrochloride and tris (beta-chloroethyl) amine hydrochloride for Hodgkin's disease, lymphosarcoma, leukemia and certain allied and miscellaneous disorders. JAMA, 1946; 132(3): 126-32
15. McKenna MK, Gachuki BW, Alhakeem SS et al: Anti-cancer activity of withaferin A in B-cell lymphoma. Cancer Biol Ther, 2015; 16(7): 1088-98

16. Shohat B, Gitter S, Abraham A, Lavie D: Antitumor activity of withaferin A (NSC-101088). Cancer Chemother Rep, 1967; 51(5): 271-76

17. Shohat B, Shaltiel A, Ben-Bassat $M$, Joshua $H$ : The effect of withaferin A, a natural steroidal lactone, on the fine structure of S-180 tumor cells. Cancer Lett, 1976; 2(2): 71-78

18. Devi PU, Sharada AC, Solomon FE: In vivo growth inhibitory and radiosensitizing effects of withaferin A on mouse Ehrlich ascites carcinoma. Cancer Lett, 1995; 95(1-2): 189-93

19. Devi PU, Kamath R, Rao BS: Radiosensitization of a mouse melanoma by withaferin A: In vivo studies. Indian J Exp Biol, 2000; 38(5): 432-37

20. Stan SD, Hahm ER, Warin R, Singh SV: Withaferin A causes FOXO3a- and Bim-dependent apoptosis and inhibits growth of human breast cancer cells in vivo. Cancer Res, 2008; 68(18): 7661-69

21. Munagala R, Kausar H, Munjal C, Gupta RC: Withaferin A induces p53-dependent apoptosis by repression of HPV oncogenes and upregulation of tumor suppressor proteins in human cervical cancer cells. Carcinogenesis, 2011; 32(11): 1697-705

22. Malik F, Kumar A, Bhushan S et al: Reactive oxygen species generation and mitochondrial dysfunction in the apoptotic cell death of human myeloid leukemia HL-60 cells by a dietary compound withaferin A with concomitant protection by $\mathrm{N}$-acetyl cysteine. Apoptosis, 2007; 12(11): 2115-33

23. Shah MA, Schwartz GK: Cell cycle-mediated drug resistance. Clin Cancer Res, 2001; 7(8): 2168-81

24. Xie X-H, Zhao H, Hu Y-Y, XI-Dong GU: Germacrone reverses Adriamycin resistance through cell apoptosis in multidrug-resistant breast cancer cells. Exp Ther Med, 2014; 8(5): 1611-15

25. Liang Y, Yang X, Li H et al: Prevalence and spectrum of BRCA1/2 germline mutations in women with breast cancer in china based on next-generation sequencing. Med Sci Monit, 2018; 24: 2465-75

26. Yi H, Wang K, Jin $\mathrm{H}$ et al: Overexpression of Rho-associated coiled-coil containing protein kinase 2 is correlated with clinical progression and poor prognosis in breast cancer. Med Sci Monit, 2018; 24: 4776-81

27. Huang S: A panel of serum noncoding RNAs for the diagnosis and monitoring of response to therapy in patients with breast cancer. Med Sci Monit, 2018; 24: 2476-88 